\title{
COVID-19 pneumonia in a patient with adult T-cell leukemia-lymphoma
}

\author{
Rika Hosoba, ${ }^{1)}$ Shinichi Makita, ${ }^{1)}$ Mika Shiotsuka, ${ }^{2)}$ Osamu Kobayashi, ${ }^{2)}$ Kiyoko Nakano, ${ }^{2)}$ \\ Mimiko Muroya, ${ }^{2)}$ Naoko Okada, ${ }^{3)}$ Makiko Suzuki, ${ }^{4)}$ Hanae Ida, ${ }^{1)}$ Suguru Fukuhara, ${ }^{1)}$ \\ Wataru Munakata, ${ }^{1}$ Tatsuya Suzuki, ${ }^{1)}$ Dai Maruyama, ${ }^{1,}$ Akiko Miyagi Maeshima, \\ Hiromichi Matsushita, ${ }^{(0}$ Noboru Yamamoto, ${ }^{7}$ Yuichiro Ohe, ${ }^{8)}$ Satoshi Iwata, ${ }^{2}$ and Koji Izutsu ${ }^{1)}$
}

\begin{abstract}
Although some patients with COVID-19 develop only mild symptoms, fatal complications have been observed among those with comorbidities. As patients with cancer are immunocompromised, they are thought to have a high risk of severe illness associated with COVID-19. We report a COVID-19 patient with adult T-cell leukemia-lymphoma (ATL) who was treated using favipiravir. A 69-year-old woman with lymphoma-type ATL was treated using cyclophosphamide, doxorubicin, vincristine, prednisolone and mogamulizumab (M-CHOP) with substantial efficacy. However, in cycle 4 of M-CHOP therapy, she developed fever with mild cough. The patient was admitted to the hospital and CT revealed bilateral ground-glass opacities. SARS-CoV-2 was detected by RT-PCR and the patient was diagnosed with COVID-19. Considering severe immunosuppression caused by ATL, we initiated favipiravir therapy. Subsequently, the fever improved without antipyretics and her C-reactive protein level decreased rapidly. SARS-CoV-2 PCR tests were negative on days 17 and 18 of favipiravir therapy, and the patient was discharged without residual disease on the final CT. This is the first documented case of COVID-19 in a patient with ATL. Although severe immunosuppression caused by ATL was present, severe COVID-19 pneumonia did not develop. The immunosuppressed condition caused by hematological malignancy may not always be a risk factor for severe illness associated with COVID-19. Further accumulation of data regarding COVID-19 in patients with hematological malignancies is warranted to clarify the risk factors for severe illness, the best-in-class antiviral agent, and the optimal treatment strategy in this population.
\end{abstract}

Keywords: adult T-cell leukemia-lymphoma, COVID-19, favipiravir, immunocompromised, SARS-CoV-2

\section{INTRODUCTION}

Coronavirus disease 2019 (COVID-19) is a severe respiratory disorder caused by severe acute respiratory syndrome coronavirus-2 (SARS-CoV-2) infection. It has spread since the first case in December 2019 in Wuhan, China, resulting in a global outbreak of COVID-19. ${ }^{1}$ Although some COVID19 patients develop only mild symptoms and recover without intervention, fatal complications, such as severe pneumonia, acute respiratory distress syndrome, and cardiac injury, have been observed, especially among the elderly or those with comorbidities. $^{2-4}$ As patients with cancer, including hematological malignancies, are immunocompromised, they are thought to have a high risk of severe illness associated with COVID-19. ${ }^{5-7}$ As there are limited data about COVID19 , the optimal standard-of-care remains undetermined.

We report the first documented case of a COVID-19 patient with adult T-cell leukemia-lymphoma (ATL) who was treated using favipiravir.

\section{CASE REPORT}

A 69-year-old woman was diagnosed with lymphomatype ATL. She had no family history of hematological

Received: July 9, 2020. Revised: August 4, 2020. Accepted: August 12, 2020. J-STAGE Advance Published: September 3, 2020

DOI: $10.3960 /$ jslrt.20030

${ }^{1}$ Department of Hematology, National Cancer Center Hospital, Tokyo, Japan, ${ }^{2}$ Office of Infection Control and Prevention, National Cancer Center Hospital, Tokyo, Japan, ${ }^{3}$ Nursing Office, Ward 11B, National Cancer Center Hospital, Tokyo, Japan, ${ }^{4}$ Nursing Office, Ward 18, National Cancer Center Hospital, Tokyo, Japan, ${ }^{5}$ Department of Pathology, National Cancer Center Hospital, Tokyo, Japan, ${ }^{6}$ Department of Laboratory Medicine, National Cancer Center Hospital, Tokyo, Japan, ${ }^{7}$ Department of Experimental Therapeutics, National Cancer Center Hospital, Tokyo, Japan, ${ }^{8}$ The COVID-19 Treatment Team, National Cancer Center Hospital, Tokyo, Japan

Corresponding author: Shinichi Makita, MD, PhD, Department of Hematology, National Cancer Center Hospital, 5-1-1 Tsukiji, Chuo-ku, Tokyo 104-0045, Japan

E-mail:smakita@ncc.go.jp

Copyright (C) 2020 The Japanese Society for Lymphoreticular Tissue Research

(c) BY-NC-SA This work is licensed under a Creative Commons Attribution-NonCommercial-ShareAlike 4.0 International License. 
malignancies, including ATL, and no past history. She was started on cyclophosphamide, doxorubicin, vincristine, and prednisolone in combination with mogamulizumab (M-CHOP) with substantial efficacy. However, on day 15 of M-CHOP cycle 4 , she developed fever of up to $38.0^{\circ} \mathrm{C}$ with mild cough and sore throat. Levofloxacin at $500 \mathrm{mg}$ once daily was administered as empiric therapy for febrile neutropenia, but fever persisted. On day 20 of cycle 4, the patient visited our hospital. Although she had mild cough and sore throat, her oxygen saturation was $98 \%$ in room air. Her peripheral white blood cell count was $2,700 / \mu \mathrm{L}$ (neutrophils, $84 \%$; lymphocytes, 4\%), hemoglobin was $11.2 \mathrm{~g} / \mathrm{dL}$, and platelet count was $27.1 \times 10^{4} / \mu \mathrm{L}$. Her CD4-positive T-cell count was low $(23 / \mu \mathrm{L})$ and her $\mathrm{C}$-reactive protein (CRP) level increased to $4.11 \mathrm{mg} / \mathrm{dL}$. Other laboratory findings, including biochemical analysis and coagulation assays, were normal (Table 1). Whole-body computed tomography (CT) revealed bilateral and subpleural ground-glass opacities typical of COVID-19 (Fig. 1A). However, lymphadenopathy was not observed and her ATL was in complete remission. The patient was admitted to the hospital and empiric therapy for bacterial (piperacillin/tazobactam) and fungal infection (voriconazole) was started. Her performance status was 1 on admission. The next day, SARS-CoV-2 was detected by real-time reverse transcription polymerase chain reaction (RT-PCR) from a nasopharyngeal swab and the patient was diagnosed with COVID-19. With no standard-of-care for COVID-19, she received only supportive care such as acetaminophen. Five days after admission, RT-PCR for SARSCoV-2 remained positive even though her fever was self-limited with supportive care. Considering the severe immunosuppression caused by ATL, we initiated favipiravir therapy at 1,600 mg twice daily (BID) on day 5 after admission, followed by $800 \mathrm{mg}$ BID. The patient provided written informed consent and emergency approval for off-label use was obtained from the Director of National Cancer Center Hospital, followed by institutional review committee approval.

After the initiation of favipiravir, the fever improved without antipyretics and her CRP level decreased rapidly (Fig. 2). On day 5 of favipiravir, follow-up CT demonstrated a decrease in the distribution of ground-glass opacities (Fig. 1B). Grade 3 transient transaminitis was observed on day 3 of favipiravir treatment. However, it may have been associated with the increased trough level of voriconazole $(14.23 \mu \mathrm{g} / \mathrm{mL})$. Grade 1 hyperuricemia, which is associated with favipiravir, was observed from day 7 , but it was manageable using oral febuxostat. On day 10 , SARS-CoV-2

Table 1. Laboratory findings at the time of admission

\begin{tabular}{|c|c|c|c|c|c|}
\hline \multicolumn{3}{|l|}{ Blood cell counts } & \multicolumn{3}{|c|}{ Biochemistry } \\
\hline WBC & 2,700 & $/ \mu \mathrm{L}$ & $\mathrm{TP}$ & 6.3 & $\mathrm{~g} / \mathrm{dL}$ \\
\hline Segmented neutrophils & 71.0 & $\%$ & Alb & 3.2 & $\mathrm{~g} / \mathrm{dL}$ \\
\hline Stab neutrophils & 13.0 & $\%$ & T-Bil & 0.5 & $\mathrm{mg} / \mathrm{dL}$ \\
\hline Lymphocytes & 4.0 & $\%$ & AST & 20 & $\mathrm{U} / \mathrm{L}$ \\
\hline Monocytes & 11.0 & $\%$ & ALT & 8 & $\mathrm{U} / \mathrm{L}$ \\
\hline Eosinophils & 0.0 & $\%$ & LDH & 204 & $\mathrm{U} / \mathrm{L}$ \\
\hline Basophils & 0.0 & $\%$ & $\mathrm{Ca}$ & 8.9 & $\mathrm{mg} / \mathrm{dL}$ \\
\hline Hemoglobin & 11.2 & $\mathrm{~g} / \mathrm{dL}$ & BUN & 14 & $\mathrm{mg} / \mathrm{dL}$ \\
\hline Hematocrit & 33.5 & $\%$ & $\mathrm{Cr}$ & 0.57 & $\mathrm{mg} / \mathrm{dL}$ \\
\hline Platelets & $27.1 \times 10^{4}$ & $/ \mu \mathrm{L}$ & UA & 3.5 & $\mathrm{mg} / \mathrm{dL}$ \\
\hline \multicolumn{3}{|l|}{ Lymphocyte subsets } & \multicolumn{3}{|c|}{ Immunology } \\
\hline CD4 absolute counts & 23 & $/ \mu \mathrm{L}$ & CRP & 4.11 & $\mathrm{mg} / \mathrm{dL}$ \\
\hline \multirow[t]{5}{*}{ CD8 absolute counts } & 37 & $/ \mu \mathrm{L}$ & $\operatorname{IgG}$ & 1277 & $\mathrm{mg} / \mathrm{dL}$ \\
\hline & & & $\operatorname{IgA}$ & 181 & $\mathrm{mg} / \mathrm{dL}$ \\
\hline & & & $\operatorname{IgM}$ & 106 & $\mathrm{mg} / \mathrm{dL}$ \\
\hline & & & KL-6 & 145 & $\mathrm{U} / \mathrm{mL}$ \\
\hline & & & SP-D & 50.9 & $\mathrm{ng} / \mathrm{mL}$ \\
\hline \multicolumn{3}{|l|}{ Infection biomarkers } & \multicolumn{3}{|c|}{ Coagulation } \\
\hline Beta-D-glucan & negative & & PT & 13.6 & $\mathrm{sec}$ \\
\hline CMV antigenemia & negative & & APTT & 36.6 & $\mathrm{sec}$ \\
\hline Galactomannan antigen & negative & & Fibrinogen & 378 & $\mathrm{mg} / \mathrm{dL}$ \\
\hline Procalcitonin & 0.15 & $\mathrm{ng} / \mathrm{mL}$ & FDP & 2.5 & $\mu \mathrm{g} / \mathrm{mL}$ \\
\hline
\end{tabular}

Abbreviations: Alb, serum albumin; ALT, alanine aminotransferase; APTT, activated partial thromboplastin time; AST, aspartate aminotransferase; BUN, blood urea nitrogen; $\mathrm{Ca}$, calcium; CMV, cytomegalovirus; Cr, Creatinine; CRP, C-reactive protein; FDP, fibrin degradation product; Ig, immunoglobulin; KL-6, sialylated carbohydrate antigen Krebs von den Lungen-6; LDH, lactate dehydrogenase; PT, prothrombin time; SP-D, surfactant protein D; T-Bil, total bilirubin; TP, total protein; UA, uric acid; WBC, white blood cell count 

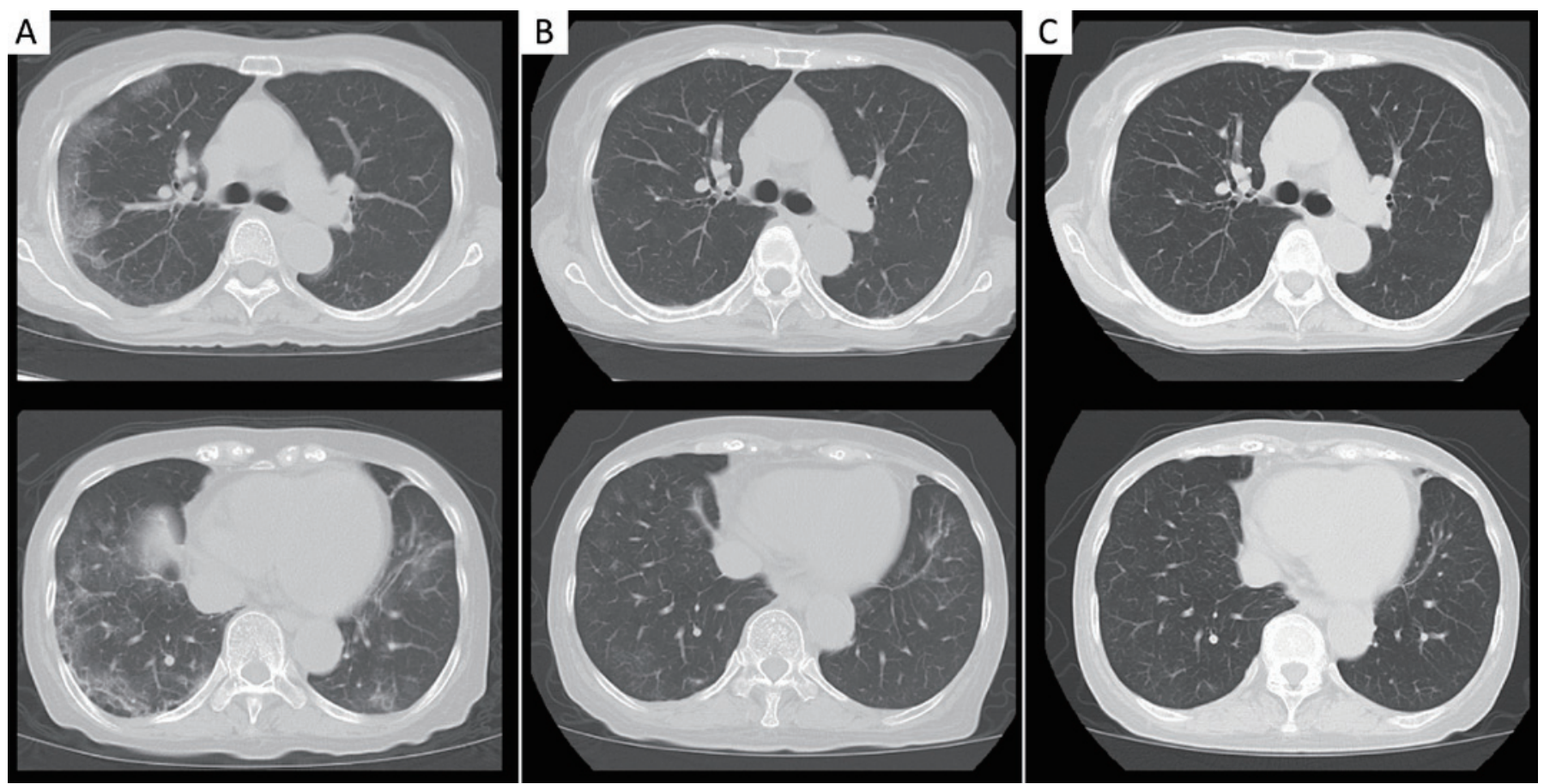

Fig. 1. Computed tomography $(\mathrm{CT})$ scans

(A) Bilateral and subpleural ground-glass opacities typical of COVID-19 are seen on admission.

(B) A decrease in the distribution of ground-glass opacities is seen on day 5 of favipiravir therapy (the $10^{\text {th }}$ hospital day).

(C) The scan on day 18 of favipiravir therapy (the $23^{\text {rd }}$ hospital day) shows resolution of disease.

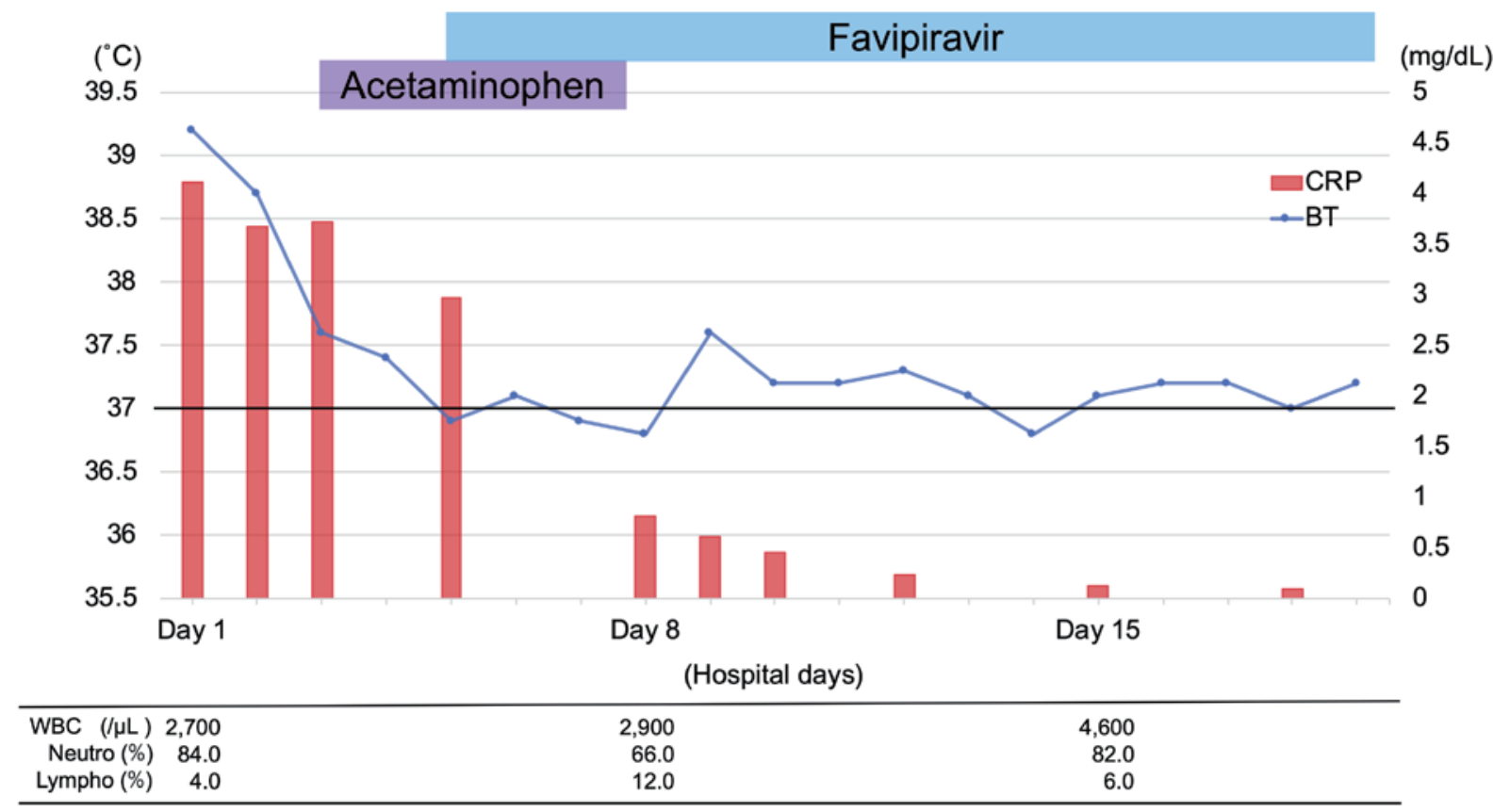

Fig. 2. The patient's clinical course and changes in body temperature

Abbreviations: BT, body temperature; CRP, C-reactive protein 
remained detectable by follow-up PCR. Therefore, we continued favipiravir until the maximum approved treatment duration of 14 days. SARS-CoV-2 PCR tests on days 17 and 18 were negative, and the patient was discharged without residual disease on the final CT on day 18 (Fig. 1C).

\section{DISCUSSION}

To the best of our knowledge, this is the first documented case of COVID-19 in a patient with ATL.

ATL is a distinct entity of peripheral T-cell lymphoma caused by human T-lymphotropic virus type-I (HTLV-1). As HTLV-1 infects CD4-positive T lymphocytes and results in oncogenesis, patients with ATL have severe immunodeficiency associated with lymphoma itself. ${ }^{8}$ In addition, low CD4 counts continue following chemotherapy against ATL. In our patient, the CD4 count at COVID-19 onset was only $23 / \mu \mathrm{L}$. Therefore, patients with ATL are thought to have a high risk of severe illness associated with COVID-19. However, the patient had mild COVID-19 infection that did not require supplemental oxygen or ICU management despite severe immunosuppression by ATL. This was partly because the patient did not have other risk factors for severe illness associated with COVID-19 such as asthma, diabetes, kidney disease, severe obesity and elderly age. Successful disease control of ATL may also have played a role in the favorable clinical course of COVID-19 in this patient. Therefore, the immunocompromised condition caused by hematological malignancies may not be a sole risk factor for severe illness in COVID-19. Currently, anti-inflammatory agents, such as tocilizumab, ibrutinib and acalabrutinib, have been tested for the treatment of COVID-19.9-12 Thus, the immunosuppressed condition may play a protective role against severe cytokine storm associated with COVID-19. Further accumulation of data is warranted to clarify the risk factors for severe COVID-19 among patients with hematological malignancies.

Favipiravir is a pyrazine analog that selectively and potently inhibits the RNA-dependent RNA polymerase (RdRp) of RNA viruses. ${ }^{13}$ It was approved by the Pharmaceutical and Medical Device Agency (PMDA) for the treatment of patients with novel or re-emerging pandemic influenza virus infection in Japan. ${ }^{14}$ As the catalytic domain of RdRp is conserved among different types of RNA viruses, favipiravir is expected to be an effective antiviral agent against SARS-CoV-2. In the present case, we decided to use favipiravir because the patient was immunocompromised, which is considered to increase the risk for severe illness associated with COVID-19, and other antiviral agents were not available in Japan at that time. COVID-19 was treated by favipiravir and it may have prevented the progression of COVID-19 to fatal pneumonia. However, we cannot conclude that favipiravir was effective against COVID-19 in this patient because the fever was self-limited with supportive care before the initiation of favipiravir. To confirm the actual efficacy of favipiravir, further evaluation in a largescale clinical trial is warranted. ${ }^{15}$ Currently, several agents other than favipiravir with three main mechanisms of action are being tested or approved for the treatment of COVID-19. These include agents that inhibit fusion of the viral envelope, such as nafamostat and camostat, ${ }^{16,17}$ agents that inhibit viral RNA duplication, such as anti-HIV agents lopinavir/ritona$\operatorname{vir}^{18}$ and remdesivir, ${ }^{19}$ and anti-inflammatory agents, including tocilizumab (an interleukin-6 receptor blocker), ${ }^{9}$ ibrutinib and acalabrutinib (Bruton's tyrosine kinase inhibitors). ${ }^{10-12}$ Further careful evaluation is required to determine the bestin-class treatment and the actual role of these drugs in the treatment of COVID-19.

In conclusion, we report a case of COVID-19 in a severely immunocompromised ATL patient. As the patient did not develop severe COVID-19 pneumonia, the immunosuppressed condition caused by hematological malignancy may not always be a risk factor for severe illness associated with COVID-19. Further accumulation of data regarding COVID-19 in patients with hematological malignancies is warranted to clarify the risk factors for severe illness, the best-in-class antiviral agent, and the optimal treatment strategy in this population.

\section{AUTHORSHIP STATEMENT}

R.H. and S.M. wrote the initial draft of the manuscript. S.M. is the attending physician of this patient. M.S. O.K. and S.I. played advisory roles in the management of COVID19 in this patient. The other authors assisted in the preparation of the manuscript. The final version of the manuscript was approved by all authors.

\section{ACKNOWLEDGMENTS}

We thank all of the health care workers, including nurses in Ward 11B and Ward 18, clinical research coordinator (Ms. Miki Ito) and doctors of the COVID-19 Treatment Team (Drs. Yuka Ahiko, Tsutomu Hayashi, Ken Masuda, Makoto Nishino, Junya Oguma, Yu Okubo, Tatsunori Shimoi and Yuki Shinno) at the National Cancer Center Hospital for managing the patient. We also sincerely thank health care workers at the frontlines in the fight against COVID-19 worldwide.

\section{CONFLICT OF INTEREST}

TS received honoraria from Chugai; DM received honoraria from Ono Pharmaceutical, Celgene, Takeda, Janssen Pharma, Chugai, BMS, Eisai, Kyowa-Hakko Kirin, Zenyaku Kogyo, Synmosa Biopharma and Nippon Shinyaku, and research grants from Ono Pharmaceutical, Celgene, Takeda, Janssen Pharmaceutical, Chugai, BMS, Merck, Amgen Astellas BioPharma, Astellas Pharma, Sanofi, Novartis and Otsuka Pharmaceutical; NY received research grants from Chugai, Taiho, Eisai, Lilly, Quintiles, Astellas, BMS, Novartis, Daiichi-Sankyo, Pfizer, Boehringer Ingelheim, Kyowa-Hakko Kirin, Bayer, Ono Pharmaceutical, Takeda, Janssen Pharma, MSD, Merck, GSK and Sumitomo 
Dainippon, and received honoraria from Ono Pharmaceutical, Chugai, AstraZeneca, Pfizer, Lilly, BMS, Eisai, Otsuka, Takeda, Boehringer Ingelheim, Cimic and Sysmex; YO received research grants from AstraZeneca, BMS, Chugai, Eli Lilly, Ignyta, Janssen Pharma, Kyorin, Nippon Kayaku, Novartis, Ono Pharmaceutical, Pfizer, Taiho and Takeda, and received honoraria from AstraZeneca, BMS, Chugai, Eli Lilly, Janssen Pharma, Kyorin, Nippon Kayaku, Novartis, Ono Pharmaceutical, Pfizer, Taiho, Takeda, Amgen, Boehringer Ingelheim and Celtrion; KI received honoraria from FujiFilm Toyama Chemical outside the submitted work. RH, SM, MiS, OK, KN, MM, NO, MaS, HI, SF, WM, AMM, $\mathrm{HM}$ and SI have nothing to disclose.

\section{REFERENCES}

1 Fauci AS, Lane HC, Redfield RR. Covid-19 - navigating the uncharted. N Engl J Med. 2020; 382 : 1268-1269.

2 Li Q, Guan X, Wu P, et al. Early transmission dynamics in Wuhan, China, of novel coronavirus-infected pneumonia. N Engl J Med. 2020; 382 : 1199-1207.

3 Richardson S, Hirsch JS, Narasimhan M, et al. Presenting characteristics, comorbidities, and outcomes among 5700 patients hospitalized with COVID-19 in the New York City area. JAMA. 2020; $323: 2052-2059$

4 Center for Disease Control and Prevention. Coronavirus Disease 2019. Groups at Higher Risk for Severe Illness. https:// www.cdc.gov/coronavirus/2019-ncov/need-extra-precautions/ groups-at-higher-risk.html. [Accessed on 30-May-2020].

5 Liang W, Guan W, Chen R, et al. Cancer patients in SARSCoV-2 infection: a nationwide analysis in China. Lancet Oncol. 2020; $21: 335-337$

6 European Society for Medical Oncology. Cancer patient management during the COVID-19 pandemic. ESMO guidelines. https://www.esmo.org/guidelines/cancer-patient-managementduring-the-covid-19-pandemic. [Accessed on 24-Apr-2020].

7 Kuderer NM, Choueiri TK, Shah DP, et al. Clinical impact of COVID-19 on patients with cancer (CCC19): a cohort study. Lancet. 2020; 395 : 1907-1918.

8 Ishitsuka K, Tamura K. Human T-cell leukaemia virus type I and adult T-cell leukaemia-lymphoma. Lancet Oncol. 2014; 15 : e517-e526.
9 Di Giambenedetto S, Ciccullo A, Borghetti A, et al. Off-label use of tocilizumab in patients with SARS-CoV-2 infection. J Med Virol. 2020 in press. doi:10.1002/jmv.25897

10 Roschewski M, Lionakis MS, Sharman JP, et al. Inhibition of Bruton tyrosine kinase in patients with severe COVID-19. Sci Immunol. 2020; 5 : eabd0110.

11 Treon SP, Castillo JJ, Skarbnik AP, et al. The BTK inhibitor ibrutinib may protect against pulmonary injury in COVID-19infected patients. Blood. 2020; 135 : 1912-1915.

12 AstraZeneca News Release. AstraZeneca initiates CALAVI clinical trial with Calquence against COVID-19. https://www. astrazeneca.com/content/astraz/media-centre/pressreleases/2020/astrazeneca-initiates-calavi-clinical-trial-withcalquence-against-covid-19.html. [Accessed on 8-Jun-2020].

13 Furuta Y, Komeno T, Nakamura T. Favipiravir (T-705), a broad spectrum inhibitor of viral RNA polymerase. Proc Jpn Acad Ser B Phys Biol Sci. 2017; 93 : 449-463.

14 Pharmaceutical and Medical Device Agency, Japan. Avigan Review Report. https://www.pmda.go.jp/files/000210319.pdf. [Accessed on 24-Apr-2020].

15 Favipiravir Observational Study Group. Preliminary report of the favipiravir observational study in Japan. http://www.kansensho.or.jp/uploads/files/topics/2019ncov/covid19_casereport_ en_200529.pdf. [Accessed on 8-Jun-2020].

16 Hoffmann M, Kleine-Weber H, Schroeder S, et al. SARSCoV-2 cell entry depends on ACE2 and TMPRSS2 and is blocked by a clinically proven protease inhibitor. Cell. 2020; 181 : 271-280.e8.

17 Yamamoto M, Matsuyama S, Li X, et al. Identification of nafamostat as a potent inhibitor of Middle East respiratory syndrome coronavirus S protein-mediated membrane fusion using the split-protein-based cell-cell fusion assay. Antimicrob Agents Chemother. 2016; $60: 6532-6539$.

18 Cao B, Wang Y, Wen D, et al. A trial of lopinavir-ritonavir in adults hospitalized with severe Covid-19. N Engl J Med. 2020; 382 : 1787-1799.

19 Huang C, Wang Y, Li X, et al. Clinical features of patients infected with 2019 novel coronavirus in Wuhan, China. Lancet. 2020; $395:$ 497-506. 